Cahiers de philosophie de l'université de

Totalitas. Aux origines d'un concept

\title{
Totalité et démocratie. Claude Lefort avec et après Marx
}

\section{Mattia Di Pierro}

\section{(2) OpenEdition \\ 12 Journals}

Édition électronique

URL : https://journals.openedition.org/cpuc/349

DOI : $10.4000 /$ cpuc.349

ISSN : 2677-6529

Éditeur

Presses universitaires de Caen

\section{Édition imprimée}

Date de publication : 31 décembre 2016

Pagination : 155-168

ISBN : 978-2-84133-842-9

ISSN : 1282-6545

\section{Référence électronique}

Mattia Di Pierro, «Totalité et démocratie. Claude Lefort avec et après Marx », Cahiers de philosophie de l'université de Caen [En ligne], 53 | 2016, mis en ligne le 01 février 2019, consulté le 31 janvier 2023.

URL : http://journals.openedition.org/cpuc/349; DOI : https://doi.org/10.4000/cpuc.349

\section{(c) (1) $\$$}

Creative Commons - Attribution - Pas d'Utilisation Commerciale 4.0 International - CC BY-NC 4.0 https://creativecommons.org/licenses/by-nc/4.0/ 


\title{
Totalité et démocratie. Claude Lefort avec et après Marx
}

\begin{abstract}
Claude Lefort fait ses premiers pas politiques et intellectuels au sein du parti trotskiste ${ }^{1}$ auquel il a adhéré sur les conseils de Maurice Merleau-Ponty, son professeur au lycée Carnot à Paris. Mais bientôt, son adhésion au parti est remise en cause par la critique du dogmatisme interne et la réalité de l'URSS dont Lefort a tout de suite une image indubitablement négative ${ }^{2}$. La scission de 1943 et la création du groupe Socialisme ou Barbarie avec Cornelius Castoriadis est la première étape d'une longue confrontation avec la réalité du régime soviétique et avec les fondements théoriques de la pensée communiste ${ }^{3}$. Ce n'est alors pas un hasard si le désaccord sur le sens de l'engagement politique et la remise en question de quelques catégories chères au socialisme - comme celle de révolution, de peuple ou de prolétariat $^{4}$ - conduisent à la séparation en 1958, d'avec Castoriadis lui-même et de quelques-uns de ses camarades de Socialisme ou Barbarie ${ }^{5}$.
\end{abstract}

1. En 1942, Lefort commence à fréquenter les cercles de la Quatrième Internationale et en particulier le CCI (Comité communiste internationaliste), un groupe politique trotskiste créé en 1943 et qui a fusionné avec le PCI en 1944. Puis, en 1943, Lefort adhère au Parti communiste internationaliste (PCI), également d'inspiration trotskiste.

2. Cf. H. Poltier, "Qu'est-ce que la pensée du politique? Une introduction au projet philosophique de Claude Lefort", Revue de théologie et de philosophie, nº 126, 1994, p.119-141.

3. En 1946, Lefort rencontre Cornelius Castoriadis, lui aussi militant dans les rangs du PCI. Tous deux créent le courant Chalieu-Montal (Montal étant le pseudonyme de Lefort à cette période) qui, d'empreinte antistalinienne, professe un marxisme non dogmatique et une vision de l'URSS comme «état ouvrier dégénéré». En 1948, Lefort et Castoriadis fondent le groupe Socialisme ou Barbarie qui, l'année suivante, crée un magazine du même nom.

4. Cf. C. Lefort, «L'expérience prolétarienne», in Éléments d'une critique de la bureaucratie, Paris, Gallimard, 1974, p. 71-97. Dans ce cadre, voir G. Labelle, «Parcours de Claude Lefort: de l'"expérience prolétarienne" de l'“l'aliénation" à la critique du marxisme», Politique et Sociétés, $34, \mathrm{n}^{\circ} 1,2015, \mathrm{p} .17-36$.

5. Lefort fait une riche description de cette période dans une interview donnée à L'Anti-Mythes en 1975. Cf. C. Lefort, «Entretien avec l'Anti-Mythes», L'Anti-Mythes, novembre 1975, p. 30, repris dans C. Lefort, Le Temps présent. Écrits 1945-2005, Paris, Belin, 2007, p. 223-260. Voir aussi, C. Lefort, "Organisation et parti», in Éléments d'une critique de la bureaucratie, p. 98-113. 
Pour Lefort, il est de plus en plus clair que "concevoir le socialisme c'était s'abîmer dans une fiction rationaliste " ${ }^{6}$, dans la croyance en une société qui s'exprime à elle-même dans un projet clair et conscient de ses causes et de ses objectifs. Cette position intimement liée à ce refus de la totalité et d'un savoir dernier, se trouve à la base de l'enseignement de Merleau-Ponty ${ }^{7}$, cœur de la formation philosophique de Lefort ${ }^{8}$.

Guidée par ces fondamentaux, sa réflexion s'oriente vers une relecture de quelques-unes des principales catégories politiques modernes, contre le marxisme, avec et au-delà de l'œuvre de Marx. Un parcours qui ne peut être compris qu'à la lumière de l'idée d'interprétation ${ }^{9}$ qui guide tout le travail lefortien et qui se fonde sur le refus d'une connaissance stable et objective. Ainsi, comme le lecteur, qui, à la lecture d'un texte, ne peut que renoncer à une position extérieure qui ferait abstraction, par exemple, de sa formation ou du contexte historique et intellectuel, de la même manière, le pouvoir, pris dans le conflit, dans la division de classe qui donne forme à la société, ne peut faire l'objet d'une vision objective, d'un projet complètement transparent ${ }^{10}$.

6. C. Lefort, «Organisation et parti», p. 240.

7. Cf. M. Merleau-Ponty, Les Aventures de la dialectique, Paris, Gallimard, 1955; du même auteur, cf. Le Visible et l'invisible, Paris, Gallimard, 1964.

8. Cf. C. Lefort, Sur une colonne absente. Écrits autour de Merleau-Ponty, Paris, Gallimard, 1978. Pour une analyse de l'entrelacement entre la pensée lefortienne et celle de MerleauPonty, cf. G. Labelle, «Maurice Merleau-Ponty et la genèse de la philosophie politique de Claude Lefort », Politique et Sociétés, 22, n 3,2003 , p.9-44. L'influence du maître dans les positions politiques lefortiennes et le rapport avec le parcours politique de MerleauPonty sont bien considérés dans S. Moyn, "Marxism and Alterity. Claude Lefort and the Critique of Totality", in The Modernist Imagination, Intellectual History and Critical Theory, W. Breckman, P. E. Gordon, A. D. Moses, S. Moyn et E. Neaman (dir.), New York, Berghahn Books, 2008, p. 99-116.

9. Lefort consacre la totalité du chapitre final de son œuvre dédiée à Machiavel à l'idée d'interprétation (Le Travail de l'œuvre. Machiavel, Paris, Gallimard, 1972). Le chapitre, nommé «L'œuvre, l'idéologie et l'interprétation» (ibid., p. 691-776), analyse le sens de la lecture et de la critique d’une œuvre. Le lecteur, affirme Lefort, «s'oublie dans le désir de laisser la parole à l'autre, mais de cet oubli, il lui faut revenir et s'entendre parler, puis renoncer à savoir ce qui lui appartient et ce qui appartient à l'autre, puis se garder d'effacer la différence dans la fiction d'une pure pensée qui se penserait déliée de l'un et de l'autre et affronter alors l'épreuve de l'interminable pour trouver dans l'inachèvement la mesure de sa parole", ibid., p. 697. Pour une analyse du concept lefortien d'interprétation, de ses implications avec la philosophie de la perception de Merleau-Ponty et avec la pensée de Derrida, cf. B. Flynn, Machiavelli. The Practice of Interpretation, in The Philosophy of Claude Lefort. Interpreting the Political, Evanston, Northwestern University Press, 2005, p. 59-79.

10. "J'ai donc dit que la question de l'interprétation impliquait déjà la question du politique. En découvrant l'illusion du point de vue de survol, qui donne à l'interprète son pouvoir, je suis amené à comprendre ce que dit Machiavel du point de vue du Prince qui, aveuglé par sa position de pouvoir, se dissimule que celle-ci s'engendre dans la division du social, qu'il est pris lui-même dans cette division », C. Lefort, Le Temps présent, p. 256-257. 
L'idéal marxien d'une humanité réappropriée, la révolution ${ }^{11}$ et la programmation d'une société nouvelle, pleinement consciente d'elle-même, unie, tombe, pour Lefort, précisément dans l'illusion du survol. Repenser la politique en critiquant et en dépassant le marxisme, mais par une confrontation continue avec l'œuvre de Marx, se révèle ainsi une nécessité, la voie pour une nouvelle réflexion de la société et de la politique, en dehors de l'illusion totalitaire.

Cependant, précisément pour éviter cette illusion, il est toujours indispensable de continuer à lire et à interroger Marx; d'autre part, «l'illusion de savoir ce qu'il en est de Marx et de s'en détacher, entretient, tout en la déplaçant, l'illusion d'un savoir dernier sur la société, l'illusion tenace d'un pouvoir à conquérir $»^{12}$.

\section{Marx et l'institution symbolique}

Le sens de cette approche est évident dans la lecture que fait Lefort de la critique marxienne de l'État. Dans l'optique de Marx, l'État n'est rien d'autre qu'un moyen de préserver les intérêts de la classe qui détient les moyens de production. Agent premier du capitalisme, l'État est une forme précise d'oppression créée par l'accumulation du capital à la base de laquelle se trouve la violence nue des dominants. Du point de vue lefortien, «ce que Marx appelle le péché originel du capitalisme désigne aussi celui de sa théorie ${ }^{13}$ qui ne réussit pas à comprendre de quelle manière la violence sur laquelle se fonde le nouveau mode de production moderne n'est pas «muette ${ }^{14}$, mais se pose à son tour à un niveau symbolique, à partir d'une représentation de la cause et de l'effet et d'un discours qui crée une cohérence entre la loi et le réel. En ignorant le niveau symbolique, Marx ne réussit pas à saisir la portée de la formation de l'État moderne qui devient, dans sa théorie, un simple produit du changement des modes de la production. Dépassant la thèse marxiste, on doit plutôt comprendre que l'État est bien plus qu'un instrument de la bourgeoisie et que, loin d'être le produit du capitalisme, il a créé les conditions de son développement.

Pour l'auteur du Capital, la représentation, complètement dominée par le réel, ne peut être considérée que comme un ensemble de phénomènes

11. Cf. A. Arato, "Lefort, the Philosopher of 1989 ", in Claude Lefort. Thinker of the Political, M. Plot (dir.), New York, Palgrave Macmillan, 2013, p.114-123.

12. C. Lefort, Le Temps présent, p. 175.

13. C. Lefort, «Esquisse d'une genèse de l'idéologie dans les sociétés modernes», Textures, 8-9, 1974, repris dans C. Lefort, Les Formes de l'histoire. Essais d'anthropologie politique, Paris, Gallimard, 1978, p. 283.

14. Ibid. 
à travers lesquels la vie de la production se donne une image de soi. Ainsi, Marx est poussé à renvoyer la dynamique entière de la transformation historique à la dimension matérielle, à la structure économique de la société. Chaque explication trouve sa résolution dans le processus productif et le pouvoir n'est que l'expression pure d'une classe dominante ${ }^{15}$. Les ordres de la loi, du savoir et du pouvoir sont déduits des rapports de production et le langage dans lequel s'articule la pratique sociale est réduit aux effets de la division capital-travail. En d'autres termes, Marx finit par réduire le discours mythologique (religieux, politique, juridique) à une production imaginaire de conflits réels, en convertissant en produits sociaux les instances de la loi et du pouvoir ${ }^{16}$.

Lefort soutient qu'au contraire une société ne peut être comprise simplement au travers de l'analyse de ses formes productives, car, de fait, elle s'organise dans un horizon symbolique précis ${ }^{17}$, dans une mise en forme qui comporte une mise en sens. Une telle dimension symbolique est visible dans la figure du pouvoir qui, loin d'être un simple instrument au service des forces sociales préexistantes, peut être décrite plutôt comme ce en quoi la société se reconnait en donnant une image d'elle-même. D'autre part, il ne pourrait être réduit à un pouvoir de fait qu'à condition d'abolir les coordonnées de l'identité sociale.

Voilà, conclut Lefort, que la séparation entre représentation et production, entre infrastructure et superstructure, apparaît en réalité comme une dissimulation.

Ces rapports ne s'agencent, ces effets ne se développent qu'en fonction de conditions que nous ne saurions mettre au registre du réel; en revanche, ce qui est nommé tel s'ouvre aux hommes, s'ordonne, devient lisible, une fois posés les repères d'une nouvelle expérience de la loi, du pouvoir et du savoir, une fois inauguré un mode de discours dans lequel certaines oppositions, certaines pratiques de fait s'avèrent - c'est-à-dire se renvoient les unes aux

15. «Ce qui nous paraît à présent marquer la limite de la pensée de Marx, c'est qu'il traite du processus de la représentation comme s'il s'engendrait dans les aventures de la coopération et de la division, comme si cette réalité se déterminait au niveau naturel du travail», C. Lefort, Les Formes de l'histoire. Essais d'anthropologie politique, p. 290.

16. Ibid.

17. Le symbolique est aussi défini par Lefort en référence à la socioanthropologie. Particulièrement importante pour lui a été la lecture de Edward Evans-Pritchard et de son ouvrage Les Nuer. Comme écrit Gilles Labelle: «Il n'y a d'humanité qu'instituée symboliquement ou par une objectivation et, dans le cas des Nuer, cette institution ou cette objectivation se donne comme médiation vaccale. L'humanité n'est pas pour les Nuer empêchée d'être par la vachité: tout au contraire, elle est par sa médiation ", G. Labelle, Parcours de Claude Lefort, p. 22. Cf. E. Evans-Pritchard, Les Nuer. Description des modes de vie et des institutions politiques d'un peuple nilote, Paris, Gallimard, 1968. 
autres, contiennent virtuellement un sens universel, en permettant un échange réglé entre l'agir et le penser ${ }^{18}$.

Cette interprétation est sans aucun doute très influencée par une lecture phénoménologique de l'œuvre marxienne guidée par l'enseignement de Maurice Merleau-Ponty ${ }^{19}$, lequel affirme l'impossibilité de mener à terme la réduction phénoménologique, dans la conscience pure de l'expérience du monde. Comme la structure et la superstructure, la société et son discours, de même l'expérience et l'interprétation d'un sujet unique ne sont pas séparables ni analysables l'un sans l'autre. On ne peut pas, nous dit le phénoménologue français, séparer parfaitement ce qui relève de notre interprétation et ce qui vient de notre fréquentation $\mathrm{du}$ monde; nous ne pouvons pas isoler les idées pures de la conscience des faits bruts de l'expérience. Le visible et l'invisible sont irrémédiablement liés; l'expérience du monde est inséparable d'une interprétation qui repose à son tour sur des élaborations ultérieures et antérieures.

Dans sa Critique de la philosophie du droit de Hegel, Marx avait tenté de montrer la «folie» du système philosophique hégélien, en dévoilant la genèse réelle de l'État et en dénonçant la pensée qui se ferme sur elle-même dans l'illusion de pouvoir conquérir la totalité en effaçant la division entre soi et l'être. Mais Marx lui-même n'a pas réussi à pousser sa critique jusqu'au bout et, cédant à la tentation de la totalité, a mis dans les mains des sciences positives cette même capacité totalisante qu'il avait critiquée et arrachée à la philosophie. Lefort veut donc suivre la voie ouverte par Marx et abandonnée par ce dernier pour comprendre d'une part, de quelle manière les faits bruts eux-mêmes, desquels le philosophe allemand croit pouvoir partir, s'appuient aussi, en réalité, sur une élaboration préliminaire sans laquelle ces faits resteraient muets; d'autre part, de quelle manière la production est intrinsèquement liée à une dimension symbolique donnée.

À coup sûr, nous transgressons de nouveau les frontières du marxisme, en rejetant l'idée que mythes et religions sont de simples fictions humaines, mais pour tenter de penser dans son sillage un modèle dans lequel le dispositif symbolique est tel que la dissimulation de la division sociale coïncide avec le pouvoir effectif de barrer la voie du changement ou de contenir son cours ${ }^{20}$.

18. C. Lefort, «Esquisse d'une genèse de l'idéologie dans les sociétés modernes », p. 282.

19. Cf. M. Merleau-Ponty, Les Aventures de la dialectique.

20. C. Lefort, «Esquisse d'une genèse de l'idéologie dans les sociétés modernes », p. 294. 


\section{Modernité et désincorporation}

La conception marxienne de la modernité est aussi le point de départ pour la pensée lefortienne sur la signification de l'État moderne et de la révolution démocratique.

Aux yeux de Marx, la société moderne est caractérisée par deux traits: l'unification du champ social, à cause de la généralisation de l'échange et de la réduction de tous les travaux concrets en travail abstrait, et la scission entre capital et travail, avec la concentration des moyens de production qui en résulte et la formation d'une masse croissante de salariés qui ne disposent que de leur propre force de travail ${ }^{21}$. À l'intérieur d'un tel cadre prend forme une simplification de l'antagonisme social ${ }^{22}$ qui conduit la société entière à se diviser en deux pôles opposés, dont la relation met en jeu l'identité du tout. Ainsi, la réalisation de ce que Marx appelle la «dépendance personnelle» de tous les acteurs sociaux est mise en œuvre et la société peut se rapporter à elle-même dans toutes ses parties. Par conséquent, «l'espace social tend ainsi à s'apparaître dans ses propres limites - et non en référence à un lieu autre depuis lequel il serait visible ${ }^{23}$. La simplification de toutes les divisions sociales, lesquelles sont subordonnées à une division générale - entre bourgeois et prolétaires -, porte en elle la dissolution des liens de dépendance personnelle, des liens du sang et des liens avec le territoire. Chaque terme de la division, niant le terme qui le contredit, renvoie directement à l'unité de la communauté.

Adhérant à l'interprétation marxienne, Lefort affirme que la caractéristique de la modernité est l'autoreconnaissance de la société, la formation d'un niveau d'intelligibilité qui repose sur l'élimination de toute transcendance, sur le retrait d'une extériorité par laquelle la société peut se reconnaître. Mais alors que Marx voit l'origine de l'idéologie moderne comme une tentative de la classe dominante de représenter l'universel, le philosophe parisien saisit une mutation symbolique, le développement d'un discours sur le social qui se crée à partir de lui-même et qui fait preuve de sa propre impossibilité 24 . Il y voit une nouvelle modalité du rapport que la société entretient avec l'énigme de sa propre institution.

Si nous tenions pour idéologie le discours qui affronte l'impossibilité de son auto-engendrement, cela signifierait que nous convertirions cette impossibilité en un fait positif, que nous croirions en la possibilité de le maitriser, que nous

21. K. Marx, Critique de l'économie politique, in Euvres, t. I, Économie, Paris, Gallimard, 1969.

22. K. Marx, Le Manifeste communiste, in Euvres.

23. C. Lefort, «Esquisse d'une genèse de l'idéologie dans les sociétés modernes », p. 295.

24. Ibid., p. 297. 
nous installerions de nouveau en un point fictif de survol de tout discours pour «voir» la division d'où il émerge, alors que le discours ne peut qu'en faire l'épreuve en lui-même ${ }^{25}$.

En dépassant l'analyse marxienne et sa confusion entre l'ordre idéologique et l'ordre symbolique, Lefort peut concevoir la modernité démocratique en tant que forme de la coexistence qui se montre à partir d'elle-même, à travers ses propres règles et qui détermine la destruction de la forme du corps politique sur laquelle s'appuyaient les sociétés prémodernes ${ }^{26}$. Ainsi disparaissent les principes stables et impérissables servant à la justification de l'ordre du monde ou de la différenciation sociale: chaque règle, reconduite entre les mains des hommes, se montre ouverte à la modification et à la critique.

Comme si la forme du Prince, explosée avec la modernité, avait répandu ces lambeaux dans la communauté, la perte de la justification transcendante mène à une désintrication des liens entre le pouvoir, le savoir et la loi ${ }^{27}$. La loi et la connaissance gagnent une pleine indépendance et, en se développant dans le mouvement même de la recherche de leurs propres fondements, elles ne sont plus localisables. Elles ne sont suspendues à rien d'autre qu'à des discours. La politique, en tant que domaine particulier, est contrainte à affronter une limite déterminée par le respect de l'indépendance des autres sphères (justice, économie, science) que les détenteurs de l'autorité ne doivent pas transgresser ${ }^{28}$. Ce qui s'impose alors, ce qui se trouve tacitement admis dans tous les domaines de la connaissance et des activités, c'est la fragmentation de la dimension communautaire et la complète autonomie de ses parties internes ${ }^{29}$.

La destructuration de l'ordre transcendant remet en jeu cette différence entre imaginaire et réel qui apparaissait impossible auparavant et porte en elle-même le problème d'une gestion du domaine mondain qui n'est plus en lien avec l'extérieur. Se manifeste alors une différence irréductible entre la conception d'unité que la société a d'elle-même et sa division réelle qui maintient ouvert le lieu du pouvoir et selon laquelle certains sont choisis pour gouverner sans toutefois incarner la communauté ${ }^{30}$. En

25. Ibid., p. 298.

26. Cf. E. H. Kantorowicz, The King's Two Bodies. A Study in Mediaeval Political Theology, Princeton, Princeton University Press, 1957 et Les Deux Corps du roi, Paris, Gallimard, 1989. De nombreuses analyses lefortiennes sur les sociétés prémodernes et sur le rôle du pouvoir dans celles-ci renvoient à l'étude de l'œuvre de Kantorowicz.

27. C. Lefort, Le Temps présent, p.741.

28. Ibid., p.742.

29. Ibid., p. 743 .

30. "Democracy, in contrast, preserves the irreducible difference between society's unified selfconception and its real division by keeping the place of power open. Some are selected to 
d'autres mots, si d'un côté la société se reconnaît comme étant unie dans un imaginaire commun, de l'autre, elle ne renonce pas à la dimension conflictuelle dont elle reste parfaitement consciente et grâce à laquelle sont continuellement remises en question, autant son unité que sa dimension imaginaire. Comme le remarque justement James D. Ingram, dans cette articulation nous pouvons lire le rapport entre une dimension normative de la politique (l'imaginaire) et une dimension proprement réaliste et conflictuelle ${ }^{31}$.

La modernité démocratique, incompréhensible dans l'analyse marxienne, se structure précisément à partir de cette dimension conflictuelle, en maintenant ouverte une continuelle différence de la société avec elle-même, une continuelle discordance entre l'unité de la communauté et ses innombrables divisions internes, entre l'imaginaire et le réel ${ }^{32}$.

\section{Les droits de l'homme}

Dans un article publié en 1980 dans la revue Libre et dont le titre est Droits de l'homme et politique ${ }^{33}$, Lefort se mesure avec la critique marxienne des droits de l'homme, à la lumière de l'expérience totalitaire du $\mathrm{XX}^{\mathrm{e}}$ siècle. La question est posée clairement: comment démontrer, contre Marx et contre la philosophie réaliste, que les droits de l'homme sont de la politique?

Encore une fois, le point de départ pour le philosophe parisien est plus que jamais réel et il trouve son origine dans le régime soviétique et dans les témoignages des dissidents ${ }^{34}$. Dans l'analyse marxienne, l'obstacle à l'émancipation humaine était représenté par la division qui émerge avec l'apparition de la société civile et la dissimulation subséquente des droits, qui cachent la division même. Mais ce qui apparaît manifeste aux yeux

rule, but they do not embody the community. This necessary gap between the imaginary and the real leads to an important insight about the relation between normativity (usually associated with the first view of politics) and conflict (usually associated with the second)", J. D. Ingram, "The Politics of Claude Lefort's Political: Between Liberalism and Radical Democracy", Thesis Eleven, no 87, 2006, p. 37.

31. "This necessary gap between the imaginary and the real leads to an important insight about the relation between normativity (usually associated with the first view of politics) and the conflict (usually associated with the second)", ibid.

32. Dans ce cadre, la lecture d'A. Caillé est intéressante en ce qu'elle met en exergue, dans la démocratie lefortienne, le Politique rendu conscient et accepté; cf. A. Caillé, La Démission des clercs. La crise des sciences sociales et l'oubli du politique, Paris, La Découverte, 1993.

33. C. Lefort, Droits de l'homme et politique, in L'Invention démocratique. Les limites de la domination totalitaire, Paris, Fayard, 1981, p. 45-83.

34. Cf. V. Kravchenko, J'ai choisi la liberté! La vie publique et privée d'un haut fonctionnaire soviétique, J. de Kerdéland (trad.), Paris, Self, 1947 et A. Soljénitsyne, L'Archipel du Goulag, Paris, Seuil, 1973. 
de Lefort est que, lorsque ces obstacles sont dépassés, lorsque les droits sont supprimés et que la société civile est éliminée, le résultat n’est pas l'accomplissement d'une complète émancipation, mais plutôt l'oppression, unie à une autre scission dans l'homme - entre patriotes et ennemis, délateurs - et dans la société - entre gouvernants et gouvernés, oppresseurs et opprimés. Aux yeux de Lefort, en effet, le totalitarisme semble se fonder sur l'abolition de l'autonomie de la société civile, sur la négation des déterminations particulières qui la composent, ainsi que sur la violation des droits humains. Bien que dans les régimes totalitaires quelques-unes des tensions soulignées par Marx - la société civile est, par exemple, intégrée dans un tissu politique - soient résolues, personne parmi ses lecteurs ne pourrait en bonne foi conclure que le totalitarisme donne la formule de ce que le philosophe définissait "émancipation humaine " ${ }^{35}$. Dans le développement d'une société totalitaire, le processus de destruction de la société civile implique un élargissement formidable de la sphère de la politique mais pas sa disparition. Sous le spectre de l'élimination de la politique demeure irrémédiablement une division de fait.

Pour Lefort, cela signifie une seule chose: l'affirmation posée dans les pages de La Question juive, que la politique et les droits humains sont les pôles de la même illusion, ne survit pas à l'épreuve des faits ${ }^{36}$.

Selon le philosophe parisien, ce qui trompe Marx c'est l'image idéologique des droits humains, de laquelle il déduit toute son analyse. C'est précisément ce profond malentendu qui rend le penseur de Trèves aveugle à ce qui, dans le texte même de la Déclaration qu'il a analysée, apparaît derrière l'idéologie. Ainsi, dans le droit à la liberté individuelle, Marx ne voit que la mise en scène de l'homme égoïste et séparé de la communauté. Il ignore par conséquent que dans l'espace public chaque action humaine lie nécessairement un sujet à ses semblables, indépendamment de la Constitution adoptée par une société37.

35. C. Lefort, Droits de l'homme et politique, p. 55 .

36. «La critique de l'individu s'exerce d'emblée dans les horizons d'une théorie de la société où se trouvent abolies la dimension du pouvoir et avec celle-ci la dimension de la loi et celle du savoir (en donnant à ce terme sa plus large acception, pour couvrir opinions, croyances, connaissances). Une telle théorie ne permet pas de concevoir le sens de la mutation historique dans laquelle le pouvoir se trouve assigné à des limites et le droit pleinement reconnu dans l'extériorité au pouvoir: cette double aventure illisible, simple signe de l'illusion", ibid., p. 62.

37. Cf. S. Moyn, «The Politics of Individual Rights: Marcel Gauchet and Claude Lefort», in French Liberalism. From Montesquieu to the Present Day, R. Geenens et H. Rosenblatt (dir.), Cambridge, Cambridge University Press, 2012, p. 291-310. L'analyse de Moyn sur les problèmes que pose la proposition lefortienne est très intéressante, mais nous ne pouvons pas nous y arrêter ici. 
Cette même cécité apparaît par rapport à l'article 11 de la Déclaration des droits de l'homme et du citoyen de 1789 qui garantit la libre communication des pensées ou des opinions et qui est délibérément omise dans la recherche marxienne ${ }^{38}$. Un oubli que Lefort ne juge pas du tout accidentel. Cet article, en effet, déclarant que «tout citoyen peut donc parler, écrire, imprimer librement, sauf à répondre de l'abus de cette liberté dans les cas déterminés par la loi ${ }^{39}$, définit clairement la liberté d'opinion, non pas en tant que liberté de l'individu-monade isolé, mais en tant que liberté de rapport. Contrairement à ce que Marx veut démontrer, cette liberté, en tant que communication, constitue le droit que l'homme a de sortir de lui-même, en se liant aux autres à travers la parole, l'écriture et une pensée qui peut être publique.

Ce que montre cet article et que le père de la théorie communiste ne réussit pas à comprendre, c'est la portée pratique de la déclaration des droits de l'homme, mais aussi et surtout de quelle façon les droits de l'homme véhiculent une nouvelle manière d'entrer en relation avec l'espace public, en nette discontinuité avec celle de l'Ancien Régime. Donc, bien au-delà de la scission entre bourgeois et citoyen, l'article 11 définit la physionomie moderne du pouvoir et la dissolution symbolique (désintrication) de ses liens avec le Savoir et la Loi $^{40}$.

Pour appréhender l'extrême nouveauté du droit moderne, affirme Lefort, celui-ci doit être lu à travers un cadre symbolique, à la lumière du phénomène de la «révolution démocratique» moderne, de la désincorporation du corps social ${ }^{41}$. Bien sûr, déjà dans l'État monarchique, qui se fondait sur une articulation complexe de droits-privilèges, le Prince était «soumis» à des droits accordés par lui-même ou ses ancêtres, qu'il

38. C. Lefort, Droits de l'homme et politique, p. 57.

39. «La libre communication des pensées et des opinions est un des droits les plus précieux de l'homme, tout citoyen peut donc parler, écrire, imprimer librement, sauf à répondre de l'abus de cette liberté dans les cas déterminés par la loi», ibid., p. 58.

40. «Disons que l'article fait entendre qu'il y a une communication, une circulation des pensées et des opinions, des paroles et des écrits, qui échappent par principe, sauf dans des cas spécifiés par la loi, à l'autorité du pouvoir. C'est de l'indépendance de la pensée, de l'opinion, en regard du pouvoir, c'est du clivage entre pouvoir et savoir, qu'il s'agit dans l'affirmation des droits de l'homme, et non pas seulement, non pas essentiellement de la scission du bourgeois et du citoyen, de la propriété privée et de la politique», ibid., p. 58-59.

41. "D'un tel point de vue, ramener le problème du droit aux termes de la critique marxiste, opposer la forme et le contenu, dénoncer le langage qui transporte et travestit les rapports bourgeois et la réalité économique qui serait au fondement de ces rapports, c'est, en ignorant cette dimension symbolique, se priver des moyens de comprendre le sens des revendications dont la finalité est l'inscription de nouveaux droits, ainsi que les changements qui s'opèrent dans la société sous l'effet de la dissémination de ces revendications et, non moins, dans la représentation sociale de la différence des modes d'existence légitimes; c'est enfin maintenir intacte l'image du pouvoir d'État, dans la conviction tenace que seule sa conquête serait la condition du nouveau ", ibid., p. 70 . 
était toutefois contraint de respecter. Ceux-ci étaient des droits acquis, différents et spéciaux (rapportés à la noblesse ou au clergé, à des villes ou à des corporations), enracinés dans le passé et qui relevaient d'une sorte de pacte sur lequel la monarchie même se fondait. Ainsi le Prince était-il soumis à ces droits seulement parce qu'il se conformait à sa nature même, à la nature de la monarchie.

D'une manière totalement différente, le droit moderne se détermine de manière autonome et incontrôlable par rapport au pouvoir, décrivant une extériorité ineffaçable. La désincorporation du savoir et de la loi ouvre l'espace pour la reconnaissance des modalités d'existence, d'activité et de communication dont les effets sont indéterminables. Précisément, ces effets peuvent prendre la figure de la revendication de droits, des droits humains.

Un nouveau point d'attache est fixé: l'homme ${ }^{42}$. En vertu d'une constitution écrite, d'une "Déclaration», le droit est présent dans chaque individu, établi dans la nature humaine qui cependant n'est rien d'autre que ce qu'elle apparaît à travers ces droits et leur énonciation. C'est sur un tel paradoxe que s'édifient les droits humains: ils sont énoncés comme des droits qui appartiennent à l'homme mais, dans le même moment, ce dernier apparaît seulement à travers eux comme celui dont l'essence est celle d'énoncer de tels droits. La réalité de ces derniers, à son tour, n'est pas transcendante, mais se trouve dans leur propre énonciation ${ }^{43}$. En ce sens, ils ne sont pas ancrés dans un homme illusoire et indéterminé, mais font référence, plutôt, à un fondement qui reste lié seulement à son exposition et qui «se donne comme intérieur à lui et, en ceci, se dérobe à tout pouvoir qui prétendrait s'en emparer » 44 . Indéterminables, "en excès» sur chaque énonciation, ces droits portent en eux l'exigence de leur propre reformulation, dans un processus qui fuit à chaque détermination du Pouvoir et à travers lequel chaque droit est nécessairement appelé à en soutenir de nouveaux. Ils se trouvent suspendus entre l'initiative des acteurs sociaux qui se mobilisent contre le pouvoir et le pouvoir même qui, à son tour, en répondant à la limitation, tâche d'interpréter à son avantage les droits déjà reconnus 45 .

C'est cette dynamique des droits humains, leur tourbillonnement désordonné, qui les définit comme les plus adaptés à la société démocratique, bien au-delà des limites de l'État de droit ${ }^{46}$. En d'autres termes, contrairement

42. Ibid., p. 65

43. C. Lefort, "La pensée politique devant les droits de l'homme», in Le Temps présent, p. 415.

44. C. Lefort, Droits de l'homme et politique, p. 67.

45. C. Lefort, «La pensée politique devant les droits de l'homme», p. 419.

46. «Une scène politique s'ouvre sur laquelle s'engage une lutte entre la domestication du droit et sa déstabilisation-récréation permanente de par l'intégration de droits nouveaux, de revendications nouvelles considérées désormais comme légitimes. N’est-ce pas, selon 
à ce dernier, la société démocratique est le théâtre d'une contestation dont l'objet ne se réduit pas à la conservation d'un pacte tacite, mais qui prend continuellement corps à travers des foyers que le pouvoir n'est pas en mesure de contrôler complètement ${ }^{47}$, donnant lieu à une histoire qui reste ouverte. Le droit de grève, comme celui de la sécurité sociale ou le droit du travail ne sont que quelques exemples de droits qui se sont développés à partir du noyau des droits humains vers des directions différentes et imprévisibles. Ces droits, donc, sont l'expression et le résultat d'une multitude d'initiatives et d'aspirations des minorités qui, même si elles ne se combinent pas dans le signe d'une même lutte, apparaissent réunies par l'efficacité symbolique de la notion de droit ${ }^{48}$.

Se manifeste à la fois dans l'irréductibilité de la conscience du droit à toute objectivation juridique, qui signifierait sa pétrification dans un corps de lois, et dans l'instauration d'un registre public où l'écriture des lois - comme écriture sans auteur - n'a d'autre guide que l'impératif continué d'un déchiffrement de la société par elle-même ${ }^{49}$.

Dans ces batailles, inspirées par les droits humains et qui font de la lutte politique un fait social total, on ne poursuit pas une solution globale du conflit à travers la conquête, la destruction du pouvoir ou le projet d'une société entièrement nouvelle ${ }^{50}$. Elles sollicitent le pouvoir à partir de foyers multiples, dans une sorte de « résistance» qui aspire à maintenir ouverte la place du possible, la division indéterminable de la société.

Politique des droits de l'homme, politique démocratique, deux manières donc de répondre à la même exigence: exploiter les ressources de liberté et de créativité auxquelles puise une expérience qui accueille les effets de la division; résister à la tentation d'échanger le présent contre l'avenir; faire l'effort au contraire de lire dans le présent les lignes de chance qui s'indiquent avec la défense des droits acquis et la revendication des droits nouveaux, en apprenant à les distinguer de ce qui n'est que satisfaction de l'intérêt ${ }^{51}$.

Au-delà de la réalité de ce "cercle vertueux» des revendications, il nous paraît intéressant de nous arrêter sur la raison pour laquelle le droit

C. Lefort, l'existence de cette contestation sans cesse renaissante, de ce tourbillon de droits, qui porte l'État démocratique au-delà des limites traditionnelles de l'État de droit?", M. Abensour, "Démocratie sauvage" et "principe d'anarchie" ", Revue européenne des sciences sociales, t. XXXI, n' 97,1993 , p. 230.

47. C. Lefort, Droits de l'homme et politique, p. 67.

48. L'intervention de Lefort est à lire aussi dans la discussion avec Marcel Gauchet; cf. M. Gauchet, «Les droits de l'homme ne sont pas une politique», Le Débat, nº 3,1980, p.3-21.

49. C. Lefort, Droits de l'homme et politique, p. 69-70.

50. Cf. A. Arato, «Lefort, the Philosopher of 1989 ».

51. C. Lefort, Droits de l'homme et politique, p. 83 . 
se présente comme la forme la plus claire d'une société indéterminable, qui se constitue dans la recherche d'elle-même, fuyant à chaque définition. Comme Abensour le suggère, c'est justement dans sa liaison avec la notion de droit que s'éclaircit l'apparition de la démocratie dans sa forme «sauvage ${ }^{52}$ : elle "désigne positivement l'ensemble des luttes pour la défense des droits acquis et la reconnaissance des droits bafoués ou non encore reconnus » 53 . À travers l'adjectif «sauvage » ${ }^{4}$, Lefort décrit l'essence même de la démocratie, un ordre social qui n'est ni apprivoisé ni apprivoisable, qui est à la recherche continuelle de ses fondements et qui se développe dans une perpétuelle contestation et revendication de droits 55 .

De cette manière, la dynamique démocratique, mise en discussion continue du fondement, lutte incessante du sujet contre le pouvoir, apparait comme le reflet du mouvement même de l'Être. Comme la démocratie lefortienne, l'énigmatique Être brut de Merleau-Ponty est l'Esprit sauvage qui crée sa propre loi, non pas parce qu'il a tout soumis à sa volonté, mais parce que, soumis à l'Être, il se réveille continuellement au contact de l'événement pour contester la légitimité du savoir constitué ${ }^{6}$. Cette référence qui n'est en rien accidentelle indique comment la lutte pour le droit est comprise dans le mouvement plus grand de la révolution démocratique, invention d'un monde sans repos ${ }^{57}$ que l'analyse marxiste n'était pas en mesure de saisir.

Mattia Di Pierro Scuola Normale Superiore di Pisa - Université Paris Diderot-Paris 7

52. Cf. M. Abensour, «"Démocratie sauvage" et "principe d'anarchie” ». Avec Lefort: «On voudrait en outre que les droits de l'homme ne soient point réduits aux droits acquis [...]; qu'il soit dit encore que la protection des libertés individuelles n'est pas seule en cause mais aussi la nature du rapport social et que, là où la sensibilité au droit se diffuse, la démocratie est nécessairement sauvage et non pas domestiquée», C. Lefort, Éléments d’une critique de la bureaucratie, p. 23 .

53. M. Abensour, " "Démocratie sauvage" et "principe d'anarchie" ", p. 229.

54. Cet adjectif est rarement utilisé par Lefort et jamais dans les essais principaux dans lesquels il s'occupe de dessiner les traits de la démocratie moderne. Mais bien qu'il soit rarement utilisé et seulement à la marge de sa conception de la démocratie, nous croyons qu'un tel adjectif, suivant la lecture de Miguel Abensour, peut bien décrire l'essence démocratique et sa dynamique de développement liées aux droits humains. Pour l'usage de l'adjectif «sauvage» dans les écrits lefortiens, cf. «La communication démocratique. Entretien avec Paul Thibaud et Philippe Raynaud», in Le Temps présent, p. 390 et Éléments d'une critique de la bureaucratie, p. 23.

55. C. Lefort, «La communication démocratique. Entretien avec Paul Thibaud et Philippe Raynaud", in Le Temps présent, p. 390.

56. «C'est l'Esprit sauvage, l'esprit qui fait sa propre loi, non parce qu'il a tout soumis à sa volonté, mais parce que soumis à l'Être, il se réveille toujours au contact de l'événement pour contester la légitimité du savoir établi », C. Lefort, Sur une colonne absente..., p. 44.

57. Cf. M. Abensour, "Démocratie sauvage" et "principe d’anarchie”», p. 230-233. 
